\title{
The crop milk: a potential new route for carotenoid-mediated parental effects
}

\author{
Cyril Eraud, Adrien Dorie, Anne Jacquet and Bruno Faivre
}

C. Eraud (correspondence), A. Dorie, A. Jacquet and B. Faivre, Université de Bourgogne, UMR-CNRS 5561, BioGéoSciences. Equipe Ecologie Evolutive. 6 Bd Gabriel. 21000 Dijon, France. Present address of C. E.: Office National de la Chasse et de la Faune Savage, Direction des Etudes et de la Recherche, CNERA Avifaune migratrice, Carrefour de la Canaudeire, 79360 Villiers-en-Bois, France. E-mail: cyril.eraud@oncfs.gouv.fr

\begin{abstract}
Allocation of carotenoid pigments, either through nutritional provisioning or from endogenous reserves, makes up a form of non-genetic parental investment to progeny that may contribute to fitness. To date, carotenoids derived from endogenous reserves have been acknowledged as important vectors in translating only avian female phenotype and environmental conditions experienced prior to laying. Here, we show that in columbidae, crop milk delivered by both parents may provide chicks with a large amount of endogenous carotenoids at the postzygotic stage. Major carotenoids were xanthophylls and beta-carotene, but their concentrations showed large variation among individuals. Interestingly, a large amount of this variation was explained by brood identity, suggesting either environmental influences and/or phenotypic influences on a parent's ability to transfer these biomolecules. Our study therefore illuminates a potential new route for endogenous carotenoid-mediated parental effects.
\end{abstract}

Non-enzymatic antioxidants such as carotenoids are key resources that can shape the development of a growing animals. They serve for several crucial functions such as the maintenance of redox homeostasis, the regulation of immune defences, the colouration of future ornaments, gene expression or the synthesis of growth factors (Hofmann and Eichele 1994, Møller et al. 2000). Therefore, early access to these biomolecules may strongly affect offspring viability and later performances.

Carotenoids are fat-soluble pigments which cannot be synthesised de novo by vertebrates but must be acquired from the diet (Olson and Owens 1998). In early life, access to this resource then relies exclusively on parental provisionning. Consequently, allocation of carotenoids makes up a form of non-genetic parental investment to their progeny that may contribute to fitness (Blount et al. 2000). In birds, parental allocation of carotenoids may occur at two distinct stages. Obviously, parents may allocate carotenoids at the postzygotic stage, through nutritional provisioning, but they may also allocate this resource earlier (at prezygotic stage) through the deposition of large amount of carotenoids in egg yolk (Blount et al. 2000, Royle et al. 2001).

If the amount of carotenoids either allocated at pre- or postzygotic stages appears to affect a large array of fitnessrelated traits (see Blount et al. 2002, 2003a, Saino et al 2003, McGraw et al. 2005, Biard et al. 2006), carotenoid pigments allocated at the prezygotic stage differ sharply from those provided through nutritional provisonning as they are directly derived from parental endogenous (maternal) resources (Blount et al. 2000). Proximate and ultimate implications of the allocation of endogenous carotenoids have therefore attracted considerable attention among evolutionary biologists, especially ornithologists, in recent years. In particular, significant advances have been made in understanding the importance of endogenous carotenoids for offspring development, as well as the proximate factors that shape the pattern of investment of this resource. For instance, studies have shown that carotenoid supply is limited for laying females and importantly, that the amount of these biomolecules invested in embryos is traded off against physiological functions related to self-maintenance (Blount et al. 2002, Saino et al., 2002). This has lead to the emergence of a widely acknowledged idea that endogenous carotenoids act as important vectors in translating female's phenotype and environmental conditions experienced prior to laying (Blount et al. 2000, 2002). However, the eventuality that endogenous carotenoid-mediated effects may arise at the postzygotic stage, and the way that male phenotype and environmental experience are implicated, have been largely neglected. The main reason for this is that in birds, egg yolk is the only known pathway for transferring endogenous carotenoids to progeny.

In some bird species including the greater flamingo Phoenicopterus ruber roseus, the emperor penguin Aptenodytes forsteri and all columbidae, chicks are fed on a secretion which is produced at the expense of parents' body fluids (Denbow 2000). In columbidae, this secretion 
is a semi-solid substance derived from the sloughed off cells of crop squamous epithelium which are engorged with nutrients transported to them by blood vessels (Bharati et al. 1997). Named "crop milk", this substance is provided by both parents and contains nutrients such as fat, proteins, minerals, enzymes and free amino acids. However, the crop milk is devoided of carbohydrates and calcium (Davies 1939), suggesting the existence of selective pathways for some nutrients that support chicks'development in early life. Hence, we may hypothesize that crop milk may also be a mean for parents to allocate endogenous carotenoids to their progeny, therefore allowing the translation of both their' phenotype and environmental conditions experienced after laying.

The presence of carotenoids in crop milk is currently unknown. The aim of the present study was therefore to investigate for the presence of such pigments in the crop milk. To achieve this, we performed biochemical analyses of crop milk dispensed to Eurasian collared dove nestlings Streptopelia decaocto. In addition, we explored potential sources of variation in carotenoid content of crop milk dispensed to chicks. More specifically, we focused on sources usually retained in studies investigating variation in egg carotenoids, such as differences among nests, seasonal variation and within brood variation.

\section{Material and methods}

\section{General field procedures for data collection}

We conducted the study on a free-living population of Collared dove in Western France $\left(46^{\circ} \mathrm{N}, 0.5^{\circ} \mathrm{W}\right)$, from Aug. to Sept. 2005. The species typically lays 2 eggs and during the first 4-5 d of life, chicks are exclusively fed on crop milk dispensed by both parents (Cramp 1985).

The study area was surveyed daily to record nest building and laying. After clutch completion, 24 nests were subsequently checked every 2-d using a mirror fixed on a telescopic cane, to determine hatching time and order. Chicks were individually marked on their right tarsus using a non-toxic permanent marker. Collecting crop milk from breeding adults is not easy since they do not store large amounts of crop milk (Bharati et al. 1997). Therefore, crop milk was obtained by performing a biopsy on 2-d-old chicks, using $5 \mathrm{ml}$ sterile syringes with 18-gauge needles. All nests were sampled at the same moment during the day (12:00-14:00) to avoid within day variation (Hôrak et al. 2004). Before performing a biopsy, the skin was carefully disinfected using $90 \%$ vol. alcohol. A small amount of crop milk $(300-500 \mu \mathrm{l})$ was then gently sucked into the syringe and transferred into a vial which was immediately kept on ice until storage at $-80^{\circ} \mathrm{C}$. We found no evidence that biopsies caused extra-mortality since all chicks fledged successfully.

\section{Preparation of crop milk and carotenoid analysis}

Crop milk samples were homogenized by vortexing with three glass beads. A small fraction $(186 \pm 20 \mathrm{mg})$ of the homogenate was then weighted $( \pm 0.01 \mathrm{mg})$ and used for carotenoid extraction. Before extraction, water was removed from the crop milk by freeze-drying samples at $-50^{\circ} \mathrm{C}$ for 12 h (Telstar, Cryodos-50). Residual material was gently pulverized, and carotenoids were extracted twice. In practice, $500 \mu \mathrm{l}$ of hexane $+0.01 \%$ of 2,6 -di-ter-butylp-cresol (to prevent degradation of carotenoids) were added to dry residuals. The mix was vortexed for $2 \mathrm{~min}$, sonicated for $5 \mathrm{~s}$ (Sun et al. 2006), and then centrifugated for $15 \mathrm{~min}$ at $1,500 \mathrm{~g}$ and $4^{\circ} \mathrm{C}$. The supernatant was removed and stored separately. This extraction procedure was repeated on the remaining residue. Supernatants from the two extractions were then pooled, and the liquid evaporated under nitrogen gas and no light. For analysis, the pigments were resuspended in $50 \mu \mathrm{l}$ of a solution of methanol and terbutyl-methyl-ether (50:50). HPLC pigment separations were performed by injecting these $50 \mu \mathrm{l}$ of pigments suspension into a 717 Waters Plus Autosampler fitted with a stainless steel ProntoSIL C18 reversed-phase column (Bischoff, Leonberg, Germany). The mobile phase was a mixture of methanol, ter-butyl-methyl-ether and deionized water delivered by a $600 \mathrm{E}$ Multisolvent pump at a constant flow rate of $1 \mathrm{ml} / \mathrm{min}$ (see Gaillard et al. 2005) for details about the mobile phase gradient). Absorption spectra were collected using a Waters 996 photodiode array detector, and chromatograms were plotted at $450 \mathrm{~nm}$. Peak identification was performed by comparing retention times and absorption properties with pure standards purchased from Extrasynthèse (Genay, France). Major carotenoid pigments were quantified using an internal standard of $\beta$-cryptoxanthin and separate calibration curves established from injection of pure standards (Gaillard et al. 2005).

\section{Statistical analyses}

Carotenoid concentration was expressed in $\mathrm{ng}$ per $\mathrm{mg}$ of fresh and dry mass of crop milk. Total carotenoid content was assessed by summing concentrations of major carotenoids. General Linear Mixed Models were used to examine the effects of nest identity, hatching order and hatching date on carotenoids concentration in crop milk samples. GLMMs were fitted with total (or specific) carotenoids concentration as the dependent variable, hatching order as a fixed factor, hatching date as a covariate, plus their interaction. To account for the lack of independence between chicks raised by the same parents, models were fitted with nest identity as a random factor. GLMMs were performed on carotenoid concentrations expressed per unit of fresh and dry milk. However, since analyses shared similar conclusions whatever the unit used, we present only results of GLMMs fitted with carotenoid concentrations in dry milk $\left(\log _{10}+1\right)$ as the dependent variable.

\section{Results}

Carotenoid pigments were detected in all crop milk samples. Mean concentrations $( \pm S E)$ were $3.81( \pm 0.37)$ and $20.50( \pm 2.48) \mathrm{ng}^{-\mathrm{mg}^{-1}}$ for fresh and dry milk, respectively. HPLC analysis revealed that xanthophylls were the major carotenoid pigments, accounting on average for $94.86 \%( \pm 1.54)$ of the total (Table 1$)$. Xanthophylls were predominantly zeaxanthin, lutein and dehydrolutein 
Table 1. Level of major carotenoids (dehydrolutein, lutein, zeaxanthin, anhydrolutein and $\beta$-carotene) detected in crop milk collected on $2 \mathrm{~d}$ old chicks of the Eurasian collared dove $(n=48)$. Means are given \pm SE.

Mean concentration $\left(\mathrm{ng} \times \mathrm{mg}^{-1}\right)$

Frequency $(\%)$

\begin{tabular}{lcrrr} 
Carotenoids & Fresh crop milk & Dry crop milk & Of occurence & Of total carotenoids \\
\hline Dehydrolutein & $0.53 \pm 0.08$ & $3.01 \pm 0.68$ & 100.00 & $13.17 \pm 1.11$ \\
Lutein & $1.02 \pm 0.12$ & $4.83 \pm 0.53$ & 93.75 & $28.35 \pm 2.11$ \\
Zeaxanthin & $2.03 \pm 0.23$ & $11.11 \pm 1.51$ & 97.92 & $51.21 \pm 2.42$ \\
Anhydrolutein & $0.05 \pm 0.01$ & $0.27 \pm 0.05$ & 62.50 & $2.13 \pm 0.46$ \\
$\beta$-carotene & $0.18 \pm 0.07$ & $1.28 \pm 0.72$ & 31.25 & $5.13 \pm 1.54$ \\
& & & & \\
Total Xanthopylls & $3.63 \pm 0.37$ & $19.21 \pm 2.25$ & & \\
Total Carotenoids & $3.81 \pm 0.37$ & $20.50 \pm 2.48$ & & \\
\hline
\end{tabular}

(Fig. 1, Table 1). The rarest carotenoid pigment was $\beta$ carotene (Table 1 ) and was detected in only $31 \%$ of samples (Table 1). Mean $\beta$-carotene concentrations across all samples were $0.18( \pm 0.07)$ and $1.28( \pm 0.72) \mathrm{ng}^{\mathrm{m} \mathrm{mg}^{-1}}$ for fresh and dry milk, respectively. When $\beta$-carotene was detected, it accounted on average for $16.42 \%( \pm 3.49)$ of total carotenoids, with an upper value reaching $23.92 \%$.

Crop milk concentrations of total carotenoids showed large variation among individual samples. Estimates ranged from 0.61 to $12.08 \mathrm{ng} \times \mathrm{mg}^{-1}$ for fresh milk, and from 2.73 to $75.61 \mathrm{ng} \times \mathrm{mg}^{-1}$ after freeze-drying. Interestingly, a significant amount of this variation was explained by brood identity (GLMM; Percent of total variance: $66.33 \%$; $\left.\mathrm{F}_{23,47}=4.77, \mathrm{P}<0.001\right)$. We found a similar effect of brood identity when performing separated analyses for each carotenoid species (GLMMs; all $F_{23,47}$ values > 4.02, all $\mathrm{P}$ values $\leq 0.001$ ), excepted for $\beta$-carotene (GLMM; $\left.F_{23,47}=1.34, P=0.254\right)$. Furthermore, concentrations of total carotenoids in crop milk collected on related siblings were significantly correlated (Spearman rank coefficient; $\left.r_{s}=0.73, P<0.001\right)$ and we found no evidences that the concentration of total carotenoids differed according to hatching rank (GLMM; $\left.F_{1,23}=2.15, P=0.157\right)$. Finally, neither hatching date nor hatching rank $\times$ hatching date interaction explained a significant amount of variation in

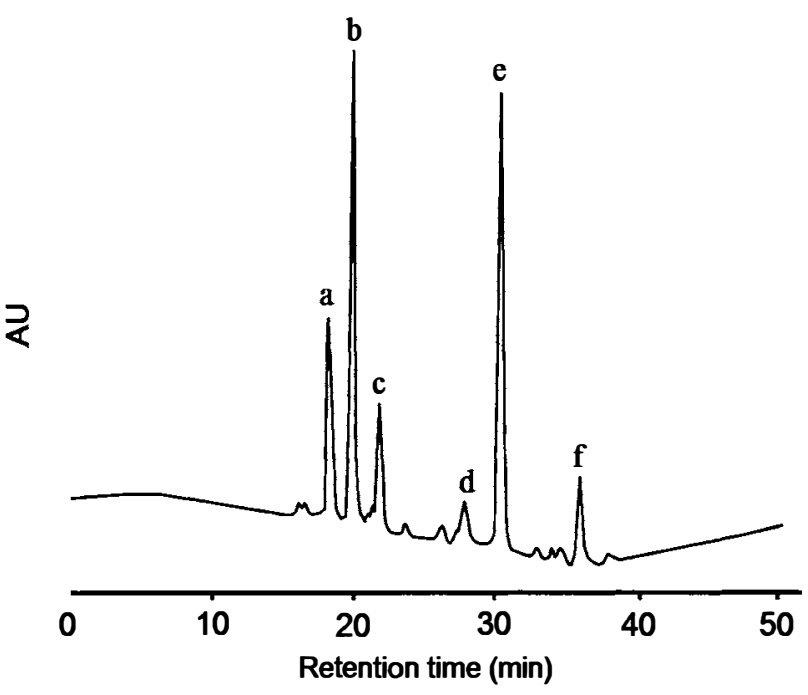

Fig. 1. HPLC chromatogram of the most abundant carotenoid pigments present in a sample of crop milk obtained from a $2 \mathrm{~d}$ old chick: a) dehydrolutein, b) lutein, c) zeaxanthin, d) anhydrolutein, e) $\beta$-crypytoxanthin (internal standard), and f) $\beta$-carotene. total or specific carotenoids (GLMMs; hatching date: all $\mathrm{F}_{1,23}$ values $<1.86$, all $\mathrm{P}$ values $>0.186$; hatching rank $\times$ hatching date: all $\mathrm{F}_{1,23}$ values $<2.59$, all $\mathrm{P}$ values $\geq 0.122$ ).

\section{Discussion}

Crop milk collected from $2 \mathrm{~d}$ old collared dove chicks contained carotenoid pigments. As crop milk is dispensed and produced by both parents at the expense of their body fluids (Denbow 2000), these results suggest that crop milk is a potential post-hatching route for carotenoidmediated parental effects. While the physiological basis of crop milk production differs from that of mammalian milk, these results parallel those reporting the presence of carotenoids in colostrum and breast-milk (Patton et al. 1990, Schweigert and Gottwald 1999, Macias and Schweigert 2001).

Several xanthophylls found in crop milk were previously shown to improve important health and fitness-related functions when transferred in large amounts into eggs, or when provided through an enriched diet. For instance in birds, exposure to large amounts of lutein and zeaxanthin were shown to improve immunological defences and sexual attractiveness (Blount et al. 2003b, Saino et al. 2003), antioxidant functions (Blount et al. 2002), post-fledging survival prospects (McGraw et al. 2005) or colouration of growing feathers (Biard et al. 2006). As precursor of vitamin $A, \beta$-carotene is also known to enhance health related functions such as resistance to infections, growth and antioxidant defences (Bendich and Olson 1989). However, carotenoid levels in fresh crop milk were sharply below those reported in eggs of bird species (Royle et al. 2001, Blount et al. 2002) including the collared dove $\left(119 \pm 5.24 \mathrm{ng} \times \mathrm{mg}^{-1}\right.$ of fresh yolk; Eraud et al. unpubl. data). It is therefore unclear how variation in crop milk carotenoids may affect offspring phenotype. Nevertheless, the daily crop milk intake by $1-5 \mathrm{~d}$ old squabs can be up to $72 \%$ of their body weight (de Cock et al. 1991), so the total amount of carotenoid intake may therefore reach important levels when summed over the first days of life. Indeed, on the basis of a daily crop milk intake of $8 \mathrm{~g}$ (Cramp 1985), we calculated that the total amount of carotenoids ingested by $1-5 \mathrm{~d}$ old collared dove chicks would reach up to $152.4 \mu \mathrm{g}$. This corresponds to about half of the total amount of carotenoids transferred into egg yolk $(\approx 300 \mu \mathrm{g}$ for a yolk weighting $2.5 \mathrm{~g}$; Eraud et al. unpubl. data). Accordingly, carotenoids provided by crop milk may 
represent a non-negligible source of antioxidants during the crucial period of early life, and may also potentially affect the carotenoid status of chicks. In support of this, human studies have shown that carotenoids transferred during the first few days of lactation have the potential to correct abnormally low plasma carotenoid concentrations in the neonate (Ostera et al. 1986). In addition, crop milk quality may have also the potential to affect carotenoid status later in life, because in birds, it has been shown that early exposure to carotenoids have long lasting effects on carotenoid accumulation in internal tissues and fluids (Blount et al. 2003a).

We found no evidence that carotenoid content of crop milk differed between related siblings, or was affected by hatching date. However, brood identity explained a significant amount of the variation among samples. Potential explanations for this finding may include differences among pairs to assimilate and incorporate carotenoids into their crop milk, or environmental heterogeneity in carotenoid resources that supply parents. Since parents were not bled, direct evidence that their carotenoid status affects crop milk content has not been presented. Nonetheless, the effect of brood identity indirectly supports the idea that the carotenoid content of crop milk may reflect the parent's environmental experience and/or phenotype. In addition, human studies have shown that the carotenoid content of breast milk reflects the carotenoid status of mothers. Indeed, beta-carotene supplementation in lactating women has been shown to increase milk beta-carotene concentration (Canfield et al. 1997, Rice et al. 1999).

To date, carotenoids derived from endogenous reserves have been acknowledged as important vectors in translating only avian female phenotype and environmental conditions experienced prior to laying. Interestingly, our results challenge this view, suggesting that in some species, endogenous carotenoids may also mediate both paternal effects and environmental conditions experienced after laying. However, it remains to be elucidated whether parental fitness may benefit from endogenous carotenoid transfer to their progeny by an additional route other than through egg yolk; and whether changes in parental carotenoid status after laying may also have the potential to improve or impair offspring performance. The extent to which crop milk carotenoid content is traded off against parental functions related to self-maintenance; and the the extent to which crop milk carotenoid content modulates the expression of fitness-related traits in offspring such as growth, antioxidant or immunological defences, then remain worthy of future investigation.

Acknowledgements - Experiments were carried out in compliance with European legal requirements and national permission (European convention, ETS no. 123, B. F. permit no. 21-CAE085, C. E permit no. 16-ONCFS). This study was funded by Office National de la Chasse et de la Faune Sauvage, Région Bourgogne, the Agence Nationale de la Recherche and European Social Funding (Research grant to C. E. and Research contract to B. F.). We are also grateful to Kevin J. McGraw for his valuable help in carotenoid identification. Earlier versions of the manuscript were improved by Michael Cherry.

\section{References}

Bendich, A. and Olson, J. A. 1989. Biological actions of carotenoids. - FASEB 3: 1927-1932.

Bharati, L., Shenoy, K. B. and Hedge, S. N. 1996. Biochemical differences between crop tissue and crop milk of pigeons (Columba livia). - Comp. Biochem. Physiol. A 116: 51-55.

Biard, C., Surai, P. F. and Møller, A. P. 2006. Carotenoid availability in diet and phenotype of blue and great tit nestlings. - J. Exp. Biol 209: 1004-1015.

Blount, J. D., Houston, D. C. and Møller, A. P. 2000. Why egg yolk is yellow. - Trends Ecol. Evol. 15: 47-49.

Blount, J. D., Metcalfe, N. B., Arnold, K. E., Surai, P. F., Devevey, G. L. and Monaghan, P. 2007a. Neonatal nutrition, adult antioxidant defences and sexual attractiveness in the zebra finch. - Proc. R. Soc. B 270: 1691-1696.

Blount, J. D., Metcalfe, N. D., Birkhead, T. R. and Surai, P. F. 2007b. Carotenoid modulation of immune function and sexual attractiveness in zebra finches. - Science 300: 125-127.

Blount, J. D., Surai, P. F., Nager, R. G., Houston, D. C., Møller, A. P., Trewby, M. L. and Kennedy, M. W. 2002. Carotenoids and egg quality in the lesser black-backed gull Larus fuscus. a supplemental feeding study of maternal effects. - Proc. R. Soc. B 269: 29-36.

Canfield, L. M., Guiliano, A. M., Neilson, E. M., Yap, A. H., Graver, E. J., Cui, H. A. and Blashill, B. M. 1997. Betacarotene in breast milk and serum is increased after a single beta-carotene dose. - Am. J. Clin. Nutr. 66: 52-61.

Cramp, S (ed.) 1985. Handbook of the birds of Europe, the Middle East and North Africa. - Oxford University Press, Oxford.

Davies, W. L. 1939. The composition of the crop milk of pigeon. - Biochem. J. 33: 898-901.

de Cock, H., Simoens, P., Gyselbrecht, C. and de Geest, J. P. 1991. Morphology of the crop and crop milk in the pigeon (Columba livia domestica). - Vlaams Diergeneesk Tijdschr. 60: 94-100.

Denbow, D. M. 2000. Gastrointestinal anatomy and physiology. - In: Whittow, G. C. (ed.). Sturkie's avian physiology. Academic Press, pp. 299-325.

Gaillard, M., Juillet, C., Cezilly, F. and Perrot-Minnot, M. J. 2005. Carotenoids of two freshwater amphipod species (Gammarus pulex and. roeseli) and their common acanthocephalan parasite Polymorphus minutes. - Comp. Biochem. Physiol. B 139: 129-136.

Hofmann, C. and Eichele, G. 1994. Retinoids in development. - In: Sporn, M. B., Roberts, A. B. and Goodman, D. S. (eds). The retinoids, biology, chemistry and medecine. Raven Press, pp. 387-441.

Hōrak, P., Surai, P. F., Ots, I. and Møller, A. P. 2004. Fat soluble antioxidants in brood-rearing great tits: relations to health and appearance. - J. Avian Biol. 35: 63-70.

Macias, C. and Schweigert, F. J. 2001. Changes in the concentration of carotenoids, vitamin $\mathrm{A}$, alpha-tocopherol and total lipids in human milk throughout early lactation. - Ann. Nutr. Metabol. 45: 82-85.

Møller, A. P., Biard, C., Blount, J. D., Houston, D. C., Ninni, P., Saino, N. and Surai, P. F. 2000. Carotenoid-dependent signals: indicators of foraging efficiency, immunocompetence or detoxification ability? - Avian Poultry Biol. Rev. 11: 137159. 
McGraw, K. J., Adkins-Regan, E. and Parker, R. S. 2005. Maternaly derived carotenoid pigments affect offspring survival, sex ratio, and sexual attractiveness in a colorful songbird. - Naturwiss. 92: 375-380.

Olson, V. A. and Owens, I. P. F. 1998. Costly sexual signals: are carotenoids rare, risky or required? - Trends Ecol. Evol. 13: 510-514.

Ostera, E., Balun, J., Winkler, R. and Porter, T. 1986. Influence of breast feeding on the restoration of the low serum concentration of vitamin $\mathrm{E}$ and beta carotene in the newborn infant. - Am. J. Obstet. Gynecol. 154: 1014-1017.

Patton, S., Canfield, L. M., Huston, G. E., Ferris, A. M. and Jensen, R. G. 1990. Carotenoids of human colostrum. - Lipids 25: 159-165.

Rice, A. L., Stoltzfus, R. J., de-Fransisco, A., Chakraborty, J., Kjolede, C. L. and Wahed, M. A. 1999. Maternal vitamin A or beta-carotene supplementation in lactating Bangladeshi women benefits mothers and infants but does not prevent subclinical deficiency. - J. Nutr. 129: 356-365.
Royle, N. J., Surai, P. F. and Hartley, I. R. 2001. Maternally derived androgens and antioxidants in bird eggs: complementary but opposing effects? - Behav. Ecol. 12: 381-385.

Saino, N., Bertacche, V., Ferrari, R. P., Martinelli, R., Møller, A. P. and Stradi, R. 2002. Carotenoid concentration in barn swallow eggs is influenced by laying order, maternal infection and paternal ornamentation. - Proc. R. Soc. B 269: 17291733.

Saino, N., Ferrari, R. P., Romano, M., Martinelli, R. and Møller, A. P. 2003. Experimental manipulation of egg carotenoids affects immunity in barn swallow nestlings. - Proc. R. Soc. B 270: 2485-2489.

Schweigert, F. J. and Gottwald, C. 1999. Effect of parturition on levels of vitamins $\mathrm{A}$ and $\mathrm{E}$ and of Beta-carotene in plasma and milk of mares. - Equine Vet. J. 31: 319-323.

Sun, T., Xu, Z. and Godber, J. S. 2006. Ultrasound assisted extraction in quantifying lutein from chicken liver using highperformance liquid chromatography. - J. Chromatogr. B 830: 158-160. 